\title{
Subjektive Theorien zu religiöser Vielfalt von katholischen Religionslehrkräften. Eine explorative Studie
}

\section{Matthias Gronover ${ }^{\mathrm{a}}$ / David Ambiel ${ }^{\mathrm{a}}$ / Ulrich Riegel ${ }^{\mathrm{b}}$ / Malte Brügge-Feldhake ${ }^{\mathrm{b}}$ / Sophia Jumpertz ${ }^{b}$ / Maximiliane Krämera / Reinhold Boschki ${ }^{a}$}

aEberhard Karls Universität Tübingen; bUniversität Siegen

Kontakt: matthias.gronover@uni-tuebingen.de

eingereicht: 25.06.2021; überarbeitet: 08.08.2021; angenommen: 27.09.2021

\begin{abstract}
Zusammenfassung: Subjektive Theorien von Religionslehrkräften spielen eine zentrale Rolle in der religionspädagogischen Theoriebildung. Zugleich stellen empirische Erhebungen subjektiver Theorien zur religiösen Vielfalt ein Desiderat der religionspädagogischen Forschung dar. Der Beitrag adressiert dieses Desiderat, indem er im Rahmen einer explorativen Interviewstudie Vorstellungen religiöser Vielfalt von Religionslehrkräften untersucht. Die inhaltsanalytische Untersuchung ergibt zwei Heuristiken, gemäß denen religiöse Vielfalt von Lehrkräften konstruiert wird. Einerseits greifen sie auf die nominalen Kategorien des Religionsdiskurses zurück, andererseits lässt sich ein vierdimensionales Modell entdecken, das religiöse Differenz entlang einer positionellen, einer stilistischen, einer kontextuellen und einer biografischen Dimension erfasst. Dieser Befund wird abschließend diskutiert.

Schlagwörter: religiöse Vielfalt, Heterogenität, Religionsunterricht, Inhaltsanalyse, Pluralitätsfähigkeit

Abstract: Subjective theories of religious education teachers play a central role in religious education theory building. At the same time, empirical surveys of subjective theories of religious diversity represent a desideratum of research in religious education. This article addresses this desideratum by examining religious teachers' conceptions of religious diversity in the context of an exploratory interview study. The content-analytical investigation yields two heuristics according to which religious diversity is constructed by teachers. On the one hand, they draw on the nominal categories of religious discourse; on the other hand, a four-dimensional model can be discerned that captures religious difference along a positional, a stylistic, a contextual, and a biographical dimension. This finding is discussed in conclusion.

Keywords: Religious Heterogeneity, Religious Education, Content Analysis, dealing with religious diversity
\end{abstract}

\section{Einleitung}

Religionsunterricht findet heute in einer weltanschaulich und religiös vielfältigen Gesellschaft statt. Diese Vielfalt schreibt sich in den Religionsunterricht ein und die Lehrkräfte müssen mit ihr umgehen. So ist es ein explizites Ziel dieses Unterrichts, seine Schülerinnen und Schüler zu einem kompetenten Umgang mit religiöser Vielfalt zu befähigen (Aslan, 2017; Die Deutschen Bischöfe, 2016; Rat der Evangelischen Kirche in Deutschland, 2014). Aus professionstheoretischer Perspektive setzt ein angemessener Umgang mit religiöser Vielfalt bei den Lehrpersonen ein angemessenes Problembewusstsein voraus. Denn subjektive Vorstellungen haben eine hohe Tragweite für das Agieren von Lehrkräften im Unterricht selbst, weil sie in Form von Überzeugungen das eigene Erkennen und Handeln leiten (Merk, 2020). Im Rahmen der religionspädagogischen Professionsforschung spielen subjektive Theorien neben entsprechenden Einstellungen und Haltungen deshalb eine prominente Rolle (Rothgangel, 2019). Die Subjektiven Theorien von Religionslehrpersonen zu religiöser Vielfalt sind jedoch erst anfänglich erforscht (z. B. Pohl-Patalong, Woyke, Boll, Dittrich \& Lüdtke, 2016). Der vorliegende Beitrag reagiert auf dieses Desiderat religionspädagogischer Forschung, indem er die Analyse von 22 Interviews beschreibt, 
in denen Lehrkräfte im Fach Katholische Religion an berufs- und allgemeinbildenden Schulen darüber Auskunft gegeben haben, was sie unter religiöser Vielfalt verstehen und wo und wie sie dieser in ihrem Unterricht begegnen. Es zeigt sich, dass Religionslehrerinnen und -lehrer religiöse Vielfalt einerseits sowohl entlang der Differenzen zwischen den Konfessionen und Religionen als auch innerhalb derselben konstruieren und dazu andererseits vor allem eine positionelle Perspektive auf Religion einnehmen. Um diesen Befund theoretisch einzubinden und angemessen darzustellen, wird im vorliegenden Beitrag in einem ersten Schritt das begriffliche Umfeld der religionspädagogischen Diskussion über religiöse Vielfalt beschrieben (2). Es folgt die Darlegung der Methode des Forschungsprojekts (3) und der empirischen Befunde (4). Im letzten Abschnitt werden diese Befunde diskutiert (5).

\section{Religiöse Vielfalt in der religionspädagogischen Diskussion}

Religiöse Vielfalt meint zunächst unterschiedliche Erscheinungsformen von Religion. Sie wird in der Religionspädagogik unter dem Begriff der Pluralität diskutiert, wobei unter religiöser Pluralität vor allem nebeneinanderstehende Bekenntnisse und Religionen verstanden werden (Nipkow, 1998, S. 29). Seit der Jahrtausendwende hat sich dieser Begriff zu einem „Konzeptbegriff mit programmatischem Anspruch" (Simojoki, 2020, S. 108) entwickelt. So weist der Index Theologicus im Zeitraum von 2000 bis 20211229 Treffer für das Stichwort „Pluralität“ und immer noch 614 Treffer für das Stichwort „religiöse Pluralität" aus. Im Pluralitätsbegriff kommt ein Doppeltes zum Ausdruck: Zum einen ist der Begriff der Pluralität rückgebunden an die Erfahrung der Vielfalt. Migrationsprozesse auf der einen und die digitale Vernetzung auf der anderen Seite haben dazu geführt, dass unterschiedliche religiöse Orientierungen und damit verbundene Lebens- und Ausdrucksweisen Tag für Tag erlebbar sind. Selbst in ländlichen Regionen gehört es heute zum Alltag, Menschen unterschiedlicher konfessioneller und religiöser Herkünfte, Bekenntnisse und Vollzüge zu treffen, ganz zu schweigen von der großen Vielfalt innerhalb der einzelnen Konfessionen und Religionen. Zum anderen zielt der Begriff der Pluralität auf die Anerkennung der Einzelerscheinungen von Vielfalt. Insbesondere religiöse Vielfalt gilt heute - bis auf wenige Ausnahmen - als legitim und bereichernd, wobei dieser Zugang von vielen Ungleichzeitigkeiten und Asymmetrien durchzogen ist (Klinkhammer \& Neumaier, 2020). In diesem Sinne spricht Friedrich Schweitzer von einem starken Begriff der Pluralität, weil die Dimension der Anerkennung von Vielfalt diese von der Erfahrungswirklichkeit in einen religionspädagogischen Bildungsauftrag übersetze. Nach dem „Übergang von der Säkularität in die Pluralität“ sei die Religionspädagogik erstens zu einer "dialogisch-offenen Haltung“ gezwungen, zweitens könne die Religionspädagogik Religion „,in allen ihren Handlungsfeldern [...] bereits vorfinde[n], und drittens bestehe damit die „Notwendigkeit, Orientierungsleistungen in der religiösen Pluralität zu ermöglichen“ (Schweitzer, 2002, S. 78). Religionspädagogisch ist dieser Orientierungsauftrag als programmatisch einzuordnen, weil im Zuge der Ausrichtung der inneren Anlage des Religionsunterrichts am Begriff der Pluralität die gesellschaftliche Wirklichkeit religiöser Vielfalt in den Anspruch übersetzt werden muss, Schülerinnen und Schüler zum einen religiöse Orientierung zu geben und zum anderen zu befähigen, respektvoll mit Menschen unterschiedlichster weltanschaulicher Standpunkte umzugehen (Englert, 2002a).

Hier setzt die weitreichende theoretische Dimensionierung religiöser Pluralität an, die Rudolf Englert vorgenommen hat, indem er zwischen positionellen und stilistischen Differenzen einerseits und kontextuellen und biografischen Differenzen andererseits unterscheidet (Englert, 2002b). In diesem Modell beziehen sich positionelle Differenzen auf Vorstellungen der Religionen „,von Gott, Leben und Welt“ (Englert, 2002b, S. 18), wie sie etwa in den Grundtexten der Religionen festgehalten sind und in den verschiedenen religiösen und weltanschaulichen Traditionen geronnen sind (Englert, 2002b, S. 19-23). Diese Positionen können sowohl von Gemeinschaften eingenommen werden wie auch von einzelnen Menschen. Dahingehend lassen sich nach Englert nicht-religiöse und religiöse Deutungen unterscheiden. Religiöse Deutungen wiederum spielen für verschiedene Religionen (inter-religiöse Differenz) ebenso eine Rolle, wie für verschiedene Konfessionen (inter-konfessionelle Differenz). Aber auch innerhalb der einzelnen 
Religionen oder Konfessionen lassen sich unterschiedliche Positionierungen beobachten (intra-religiöse bzw. intra-konfessionelle Differenz). Letzteres wird im Katholizismus bspw. veranschaulicht durch Vertreterinnen der Maria 2.0-Bewegung und Anhänger der Piusbrüder.

Allerdings lässt sich religiöse Pluralität nach Englert nicht nur durch positionelle Differenzen beschreiben, sondern auch entlang der "formalen Typik ihrer Realisationsstile“ (Englert 2002b, S. 18). Solche stilistischen Differenzen erfassen die Art und Weise, wie Religiosität ausgedrückt wird (Englert 2002b, S. 23-27). Das kann nach Englert exklusiv sein und damit nur die Wahrheitsansprüche und Vollzüge der eigenen Tradition anerkennend. Oder individualisiert, indem sich eine Person aus den unterschiedlichen Traditionen passende Versatzstücke herausgreift und diese in seine Anschauung aufnimmt. AuBerdem benennt Englert den säkularen Typ, der nichts mit Religion anfangen kann. Alle drei Stile stehen für eine bestimmte Art und Weise, mit Religion umzugehen und stehen somit quer zu den positionellen Differenzen.

Positionelle und stilistische Differenzen werden durch kontextuelle und biografische Differenzen als „intermittierende Faktoren“ (Englert, 2002b, S. 19) bedingt. Dabei stehen kontextuelle Differenzen für kohortenspezifische Unterschiede in der Religiosität, wie sie bspw. durch typische Sozialisationsbedingungen (etwa in der Diagnose einer Generation Y) eines Geburtenjahrgangs, des Geschlechts oder sozialen Milieus geprägt sind (Englert, 2002b, S. 27-30). Kontextuelle Differenzen beziehen sich also auf den Erfahrungskontext der Menschen und wie dieser auf deren Religiosität abfärbt. Biografische Differenzen erfassen dagegen die spezifischen Lebenslagen in der Vergangenheit und wie diese auf die eigene Religiosität Einfluss nahmen und nehmen (Englert, 2002b, S. 30-31). Das betrifft vor allem die individuelle Verarbeitung von Erlebnissen, die für die eigene Religiosität von Bedeutung sind und diese kann nach Englert entweder inhaltlich-hermeneutisch oder formal-strukturell gegliedert werden.

Diese Begriffsbestimmung ist für die Analyse religionspädagogischer Vielfalt weiterführend, weil damit ein mehrdimensionales Modell von Vielfalt gegeben wird, in dem abhängig von der eingenommenen Dimension unterschiedliche Facetten des religiösen Spektrums erfasst werden können. Religiöse Vielfalt muss nicht mehr vor allem nach religiöser Zugehörigkeit konstruiert werden (Nipkow, 1998). Vielmehr kann die Unterscheidung von Position und Stil deutlich machen, dass die Ehrfurcht vor Gott ein gemeinsames Gut zwischen jüdischen, muslimischen und christlichen Menschen ist (Stil), auch wenn sie unterschiedlichen Religionen angehören (Position). Zweitens setzt Englert die vier Dimensionen in ein theoretisches Verhältnis zueinander: Kontextuelle und biografische Differenzen bedingen als intermittierende Faktoren die Ausprägung von positionellen und stilistischen Differenzen. Drittens übersetzt Englert den empirischen Bezug religiöser Vielfalt in Orientierungen für die innere Anlage des Religionsunterrichts. Dabei sieht er in der oben genannten „markanten Pluralität“ eine Möglichkeit, „den Sinn für die in konfessionellen Bezügen steckende, bildende Kraft' zu wecken“ (Englert, 2002a, S. 91). Dieser Hinweis scheint im Zusammenhang mit Subjektiven Theorien von Religionslehrkräften besonders wichtig, denn sie übersetzen diese bildende Kraft in konkreten Unterricht.

Allerdings wurde der Pluralitätsbegriff in den letzten Jahren auch kritisiert, weil er die Machtverhältnisse, die in religiöser Vielfalt stets mitspielten, nicht erfassen könne. Letzteres verspräche der Begriff der Heterogenität, der als Ersatz für den Pluralitätsbegriff vorgeschlagen wird (Grümme, 2017). Der Heterogenitätsbegriff schließt nicht nur an eine breite pädagogische Diskussion an (Trautmann \& Wischer, 2011), sondern beinhaltet aufgrund der Tatsache, dass er sich nicht dem Religionsdiskurs verdankt, auch einer Offenheit gegenüber unterschiedlichen Phänomenen von Differenz. Wie oben gezeigt, nimmt der Begriff der Pluralität vor allem weltanschauliche Vielfalt in den Blick, während der Heterogenitätsbegriff religiöse Vielfalt zusätzlich in körperlicher, sozialer, ethnischer, geschlechtlicher, intellektueller, u. s. w. Hinsicht diskutieren kann. So bekäme man auch unterschiedliche Machtverhältnisse in den Blick, was für die Diskussion der Bildungsgerechtigkeit entscheidend sei (Grümme, 2014). 
Gleichzeitig öffnet der Heterogenitätsbegriff den Blick auf die kulturelle Verwobenheit religiöser Vielfalt und ist damit in der Lage, ein essentialistisches Verständnis dieser Vielfalt zu unterlaufen. Gerade die nicht-religiösen Dimensionen, die religiöse Vielfalt mitkonturieren, verweisen darauf, dass Religion nicht für sich genommen vorliegt, sondern immer in der jeweils konkreten und situationsspezifischen Rezeption Einzelner oder von Gemeinschaften. Auch der Begriff der Heterogenität wird zur Analyse religionspädagogischer Praxis herangezogen (Büttner, Mendl, Reis \& Roose, 2018; Reis, 2021) und orientierend-normativ mit der Zielperspektive einer „aufgeklärten Heterogenität“ verwandt (Grümme, 2018).

Es ist noch zu früh, um die innovative Kraft des Heterogenitätsbegriffs für die Diskussion religiöser Vielfalt seriös abschätzen zu können. So ließe sich etwa fragen, ob nicht die kontextuellen und biografischen Differenzen nach Englert genau das leisten könnten, was durch die Offenheit des Heterogenitätsbegriffs als dessen Mehrwert behauptet wird. Einen wichtigen Impuls stellt definitiv seine Sensibilität für Ungleichheiten und Ungerechtigkeiten dar (Grümme, 2017, S. 17), wobei einschränkend festgehalten werden kann, dass derartige Beobachtungen im Bereich des interreligiösen Lernens auch ohne Bezug auf diesen Begriff diskutiert werden (z. B. Meyer, 2019, S. 172-193; Unser, 2019). Es liegt jedoch in der Dynamik des Heterogenitätsbegriffs, dass sich die Unterschiede verschiedener Kategorien „gegenseitig beeinflussen, vorantreiben, dynamisieren oder auch relativieren" (Grümme, 2017, S. 51). Hier liegt ein zentraler Beitrag dieses Begriffs zur religionspädagogischen Diskussion um religiöse Vielfalt. In der Summe kann wohl konstatiert werden, dass sich sowohl der Pluralitäts- als auch der Heterogenitätsbegriff analogen Phänomen widmen, allerdings in unterschiedlichen theoretischen Konstellationen (Schweitzer, 2021). So bezieht sich der Pluralitätsbegriff stärker auf einen religionsbezogenen Diskurs und stellt religiöse Vielfalt damit in die Mitte seiner Überlegungen. Der Begriff der Heterogenität betont dagegen Vielfalt im allgemeinen Sinn und betrachtet Religion als ein Bestimmungsmerkmal von Vielfalt unter mehreren, bindet Religion damit aber gleichzeitig in das vielstimmige Gewebe vielfaltserzeugender Dimensionen ein. Während also der Pluralitätsdiskurs eher die Innenperspektive auf religiöse Vielfalt stark macht, bezieht sich der Heterogenitätsdiskurs stärker auf die Außenperspektive (Schweitzer, 2021, S. 34-35).

Inwieweit die religionspädagogische Diskussion um religiöse Vielfalt von den Lehrpersonen rezipiert wird, kann gegenwärtig kaum seriös abgeschätzt werden. Zwar gibt es einen breiten Forschungsstrang zu den Subjektiven Theorien von Religionslehrpersonen (z. B. Kindermann \& Riegel, 2016; LehnerHartmann, 2014), das Verständnis religiöser Vielfalt als solcher spielte bislang aber nur in der ReVikoRStudie aus Schleswig-Holstein eine zentrale Rolle (Pohl-Patalong et al., 2016). Auch die vielfältigen Untersuchungen anlässlich vorfindlicher religiöser Vielfalt im Klassenzimmer beziehen sich nur am Rand explizit auf das Verständnis der Lehrpersonen von religiöser Vielfalt. Zieht man die ReVikoR-Studie heran, zeigt sich, dass religiöse Vielfalt stark an nominalen Kategorien der Religionszugehörigkeit festgemacht wird (Pohl-Patalong et al., 2016, S. 29-39). So wird diese Vielfalt verstanden als Präsenz unterschiedlicher Religionen, christlicher Konfessionen oder ethnischer Herkünfte. Diese Herangehensweise deckt sich deutlich mit dem älteren Pluralitätsdiskurs. Allerdings finden sich auch die beiden Verständnisse über die (nicht-)religiöse Einstellung und die individuelle Glaubensvorstellung in der Stichprobe. Sie gehen zwar über ein rein nominales Verständnis religiöser Vielfalt hinaus, scheinen aber in ihren Analogien zu Englerts positioneller und stilistischer Dimension ebenfalls näher am Pluralitäts- als am Heterogenitätsdiskurs zu liegen. Letztlich fehlen jedoch hinreichend empirische Informationen, um verlässlich bestimmen zu können, was die Subjektiven Theorien von Lehrpersonen zu religiöser Vielfalt konturiert. Auf dieses Forschungsdesiderat reagiert dieser Beitrag, indem er anhand eines Samples aus Baden-Württemberg und Nordrhein-Westfalen die Forschungsfrage beantwortet, was die zentralen Kategorien der Vorstellungen von Religionslehrerinnen und -lehrern zu religiöser Vielfalt sind. 


\section{Methode}

Dieser Beitrag bezieht sich auf die problem-zentrierten Interviews, die im Rahmen eines größeren Projekts zur Rolle religiöser Heterogenität im katholischen Religionsunterricht entstanden sind. Er folgt damit einem qualitativen Design, das theoriegenerierend bzw. -differenzierend angelegt ist. Insbesondere wegen seines charakteristischen Wechsels von induktiven und deduktiven Momenten (Witzel, 2000) eignet sich das problem-zentrierte Interview für die Erhebung Subjektiver Theorien, denn seine induktiven Phasen ermöglichen es den Interviewten, ihre eigenen Zugänge zum Thema zu entfalten, während es durch seine deduktiven Phasen garantiert, dass der Bezug zur wissenschaftlichen Diskussion dieses Themas gewahrt bleibt. Analog zum ReVikoR-Projekt (Pohl-Patalong et. al., 2016, S. 21) wurden auch für diese Studie Religionslehrpersonen in einem Leitfaden-Interview befragt. Nach Informationen zum Sinn und Zweck der Untersuchung waren die Interviewten eingeladen, eine konkrete Situation aus ihrem Religionsunterricht zu erzählen, in der religiöse Vielfalt in ihren Augen eine zentrale Rolle gespielt hat. Nach dieser Erzählung wurden in einer immanenten Phase Verständnisfragen zur Erzählung geklärt und das in die Erzählung leitende Verständnis religiöser Vielfalt explizit herausgearbeitet. Dies geschah durch ad-hoc Fragen, die sich an den erzählten Inhalten orientierten und gleichzeitig das im Leitfaden aufgelistete Erkenntnisinteresse im Auge hatten. Eine Phase mit exmanenten Fragen ordnete dieses Verständnis in den größeren Kontext der Diskussion religiöser Vielfalt ein, indem auch Aspekte dieser Vielfalt angesprochen wurden, die bislang noch keine Rolle gespielt haben. Für diesen exmanenten Teil des Interviews orientierte sich der Leitfaden am oben beschriebenen Modell Rudolf Englerts, insofern zwischen positionellen, stilistischen, kontextuellen und biografischen Aspekten unterschieden wurde. Insofern entlang der letzten beiden Dimensionen auch Kategorien wie Geschlecht, Herkunft, Milieu, o. Ä. erfragt wurden, bleibt diese Operationalisierung anschlussfähig an den Heterogenitätsbegriff. Die theoretische Struktur hingegen, die Englert seinem Modell einschreibt, wurde nicht in den Leitfaden übernommen. Alle vier Aspekte stehen hier gleichgewichtig nebeneinander und es lag an den Interviewten, diese vier Dimensionen in ein Verhältnis zueinander zu setzen. Exemplarisch seien hier drei Fragen aus dem Leitfaden genannt, die sich auf unterschiedliche Facetten religiöser Vielfalt beziehen: „An welchen Merkmalen machen Sie religiöse Heterogenität fest?", „Auf welchen Ebenen zeigen sich Unterschiedlichkeiten in Hinblick auf Religion?" und „Inwiefern spielt Geschlecht, Alter, Umfeld/ Milieu, Herkunft, etc. eine Rolle?“ Natürlich wurden diese Fragen in den Interviews nicht einfach vorgelesen, sondern situationsgerecht eingespielt.

Die Interviews wurden digital aufgenommen und im Anschluss transkribiert, anonymisiert und in einem mehrstufigen Verfahren einer qualitativen Inhaltsanalyse unterzogen (Mayring, 2000) und zwar in ihrer formal-strukturierenen Version (Schreier, 2014). (i) Dazu wurde das Interviewmaterial zunächst in Segmente gegliedert, wobei jedes Segment für eine in sich geschlossene Themeneinheit steht. Eine solche Einheit kann die gesamte Erzählung eingangs des Interviews sein, stellt aber in den meisten Fällen kurze Sequenzen aus einem Satz oder zweien dar. Insgesamt wurden so 2241 Segmente identifiziert. (ii) Diese Segmente wurden paraphrasiert, zusammengefasst und kodiert. Auch wenn diese Codes induktiv entstanden sind, wurde darauf geachtet, dass inhaltlich vergleichbare Segmente mit demselben Code markiert und die verwendeten Codes, wo es inhaltlich nahe lag, an den Kategorien des Leitfadens ausgerichtet wurden. Um der Komplexität mancher Segmente gerecht zu werden, konnten einem Segment mehrere Codes zugewiesen werden. (iii) Insofern die Codierung der Interviews zwei Mitarbeiter übernahmen, konnten die Codierungen der einen Person durch die andere gegengelesen und im Fall der Fälle diskutiert werden. ${ }^{1}$ In diesem iterativen Prozess von Codierung und Diskussion entstand ein kommunikativ validiertes Codesystem. (iv) Anschließend wurden die Codes miteinander verglichen und zu Kategorien zusammengefasst, die für einen charakteristischen Zugang zu religiöser Vielfalt ste-

\footnotetext{
${ }^{1}$ An dieser Stelle wollen wir uns bei Johannes Hammer und Sarah Schlicht für ihr Engagement in der ersten Auswertungsphase der Interviews bedanken.
} 
hen. Diese Kategorisierungen entstanden im Dialog der beiden Mitarbeiter und wurden zusätzlich in vielfachen Zoom-Sitzungen im gesamten Forschungsteam des relHet-Projekts diskutiert. (v) Schließlich wurden die Kategorien daraufhin gesichtet, welche theoretischen Bezüge sich zwischen ihnen feststellen lassen. In dieser Analyserunde wurde deutlich, dass man besagte Kategorien entlang zweier Systematiken anordnen kann, die zwei zentrale Heuristiken in den Subjektiven Theorien von Religionslehrpersonen zu religiöser Vielfalt repräsentieren.

Dieser Befund stammt von einem Sample von 22 Lehrpersonen, die Religion an berufs- oder allgemeinbildenden Schulen in Baden-Württemberg oder Nordrhein-Westfalen erteilen. Die Befragten sind zwischen 32 und 52 Jahren alt. Es befinden sich 9 Frauen und 13 Männer in der Stichprobe. Die Interviews wurden im Zeitraum von Herbst 2018 bis Frühjahr 2019 geführt und dauerten zwischen 45 und 60 Minuten.

\section{Befunde}

Im Folgenden werden die beiden zentralen Heuristiken beschrieben, entlang derer die von uns Befragten religiöse Vielfalt in den Interviews konstruieren. Es handelt sich hierbei um eine Darstellung von Differenzen entlang nominaler, religionsbezogener Kategorien (4.1) und um die Darstellung von religiöser Vielfalt entlang positioneller, stilistischer, kontextueller und biografischer Aspekte (4.2).

\section{I Religiöse Vielfalt als Differenz entlang nominaler, religionsbezogener Kategorien}

Im Religionsdiskurs stellen „Religion“ und „Konfession“ (bzw. „Strömung“ oder „Glaubensgruppe“ etc.) zentrale Kategorien dar, die das religiöse Feld gruppieren. Es handelt sich hierbei um nominale Kategorien. Die Differenzen, die sich in der Gegenüberstellung dieser Kategorien ausdrücken, nutzen die Befragten häufig, um religiöse Vielfalt zu beschreiben. Im Detail lassen sich in dieser Hinsicht sechs charakteristische Konstellationen identifizieren, nämlich inter-religiöse, inter-konfessionelle, intra-religiöse, intra-konfessionelle, trans-religiöse und unbestimmte Differenzen.

Bei inter-religiösen Differenzen werden die klassischen Religionsgruppen herangezogen, um religiöse Vielfalt zu rekonstruieren. Die einzelnen Religionen werden dabei als in sich (weitgehend) homogene Gruppen verwendet, die sich untereinander unterscheiden bzw. als Identifikationskategorie für eine Unterscheidung herangezogen werden können. Im folgenden Beispiel wird eine derartige Differenz didaktisch genutzt.

Inter-religiös

"Und ich lese regelmäßig in der Weihnachtsstunde die Weihnachtsgeschichte im Koran statt in der Bibel, weil das natürlich die christlichen Schüler ein bisschen herausfordert, noch mal das abzugleichen mit dem, was sie in Erinnerung haben. Aber die muslimischen Schüler haben so auch die Möglichkeit anzudocken. Und also das war sicher so eine Situation, wo ganz deutlich wurde, wo die noch einmal Gelegenheit hatten, ihre Sichtweise einzubringen. Und die haben es auch dankbar angenommen und kamen noch einmal miteinander ins Gespräch über Dinge, ja, im Verhältnis zur Religion und so" (TUE_007_maxqda, Pos. 03).

Der Kollege weckt durch den bewussten Rekurs auf die Differenz zwischen Christentum und Islam Interesse in der Lerngruppe. Die inter-religiöse Differenz wird dabei eingesetzt, um bei den Schülerinnen und Schülern eine kognitive Dissonanz zu erzeugen (christliche Weihnachtsgeschichte im muslimischen Koran). Darüber hinaus stößt diese Differenz Identifikationsprozesse an, denn die christlichen Lernenden werden durch sie „herausgefordert“ und die muslimischen Lernenden haben die Möglichkeit, sich im Unterricht ernstgenommen zu fühlen („,andocken“, „,einzubringen“). Kern dieser Inszenierung ist die inter-religiöse Differenzierung zwischen Christentum und Islam.

Eine vergleichbare Differenzierung findet sich in den Interviews auch innerhalb der einzelnen Religionen. Meistens geht es dabei um die Gegenüberstellung von Katholischem und Evangelischem, weshalb 
diese Kategorie inter-konfessionell genannt wird. Wiederum werden beide Konfessionen als in sich (weitgehend) homogene Gruppen gedacht, die sich gegenseitig unterscheiden.

„Mir geht es auch darum, dass sie sich Gedanken machen, dass Religion in der Gesellschaft vorkommt [...] und da kommt dann auch der Punkt irgendwann mal zu sprechen, welchen Namen habe ich, was bedeutet mein Name. [...] Interessanterweise kann man gar nicht mehr differenzieren, dass die Katholiken nach Heiligen benannt sind und die Protestanten nach biblischen Namen. Das hält man gar nicht mehr durch. Das wird gar nicht mehr durchgehalten wahrscheinlich schon in den letzten zwanzig Jahren nicht mehr" (SIE_001_maxqda, Pos. 80).

Im Beispiel wird mit den beiden großen christlichen Konfessionen eine bestimmte Praxis der Namensgebung verbunden. In charakteristischer Weise wird suggeriert, es hätte eine Zeit gegeben, in der alle Katholiken nach Heiligen und alle Protestanten nach Bibelfiguren benannt worden wären. Es wird also eine Differenz zwischen den Konfessionen ausgesagt, während Unterschiede innerhalb dieser Konfessionen keine Rolle spielen. Derartige inter-konfessionelle Differenzen finden sich immer wieder in den Interviews.

Allerdings trifft man auch Interviewpassagen an, in denen eine Religion oder eine Konfession als generisches Ganzes dargestellt wird, innerhalb dessen sich Unterschiede zeigen. In diesen Fällen handelt es sich somit um intra-religiöse bzw. intra-konfessionelle Differenzen. Ein Beispiel für den ersten Fall ist die folgende Feststellung:

"In der Berufsschule haben wir Moslems. [...] Da ist es tatsächlich aber eher abhängig davon, woher die kommen. Also deutsche Moslems haben genauso wenig, können genauso wenig sagen über ihren Glauben wie die deutschen Christen. Allerdings, wenn wir Flüchtlinge haben, für die spielt der Glaube, der lebt noch. Da wird dann auch ganz klar erklärt" (TUE_004_maxqda, Pos. 30).

Die Kollegin resümiert über die muslimischen Schülerinnen und Schüler in ihrer Schule. Im Unterschied zu den bisher berichteten Fällen werden dabei Muslime nicht einer anderen religiösen Gruppe gegenübergestellt, sondern es werden Unterschiede innerhalb dieser Gruppe berichtet. Im Beispiel geht es dabei um die Auskunftsfähigkeit der jungen Erwachsenen, die in der Wahrnehmung der Kollegin in Abhängigkeit von der Herkunft unterschiedlich ausgeprägt ist. Es handelt sich somit um eine intra-religiöse Differenz. Derartige Differenzen finden sich auch auf Ebene der Konfessionen.

"Also ich glaube aber fast, bei so Sachen haben es die Evangelischen ein bisschen schwieriger, weil sie haben ja ganz häufig diese, also gerade hier in unsere Region, so ich sage jetzt in Anführungsstrichen 'hardcore Freikirchler' sitzen. Und sie haben mitunter schwieriger zu kämpfen, wenn sie mit historisch-kritischer Exegese anfangen oder so, dann fallen sie alle reihenweise vom Stuhl“" (SIE_008_maxqda, Pos. 35).

Der Kollege beobachtet beim evangelischen Religionsunterricht, dass hier die Lehrkräfte „schwieriger zu kämpfen“ haben, sofern freikirchlich organisierte Schülerinnen und Schüler mit im Unterricht sitzen. In der Logik der Aussage werden somit freikirchliche Evangelische von denen unterschieden, die landeskirchlich eingebunden sind. Es handelt sich dabei um eine Differenz innerhalb der evangelischen Tradition.

Speziell mit Blick auf die Lerngruppe wird von den Befragten immer wieder eine Unterscheidung aufgemacht, die den Bereich des Religiösen überschreitet und zwischen religiös und nicht-religiös trennt. Insofern der Religionsdiskurs der bisherige Bezugspunkt der Kategorienbezeichnungen ist, nennen wir diese Differenzierung trans-religiös.

„Hier ist es aber ja tendenziell so, dass wir halt sehr, sehr große Katholikenklassen hier haben. [...] Ich glaube, dass der Anteil von den Atheisten, also von den Leuten, die nichts sind, das sind Atheisten, das ist mal so hingestellt sage ich mal, die Leute, die eigentlich nicht getauft sind, dass der zunimmt" (SIE_001_ maxqda, Pos. 158).

Im Beispiel rechnet der prognostische Blick mit einem steigenden Anteil von „Atheisten“ im Religionsunterricht. Dabei scheint der Begriff der Atheisten weniger eine religionskritische Haltung zu bezeich- 
nen als vielmehr für die Unbeholfenheit zu stehen, wie man diejenigen nennen soll, "die nichts sind“ bzw. „die eigentlich nicht getauft sind.“ Dabei erscheinen zwei Beobachtungen bemerkenswert zu sein. Erstens werden nicht-religiöse oder säkulare Schülerinnen und Schüler in der Regel als eine in sich homogene Gruppe kommuniziert. Die weltanschauliche Vielfalt innerhalb des säkularen Feldes spielt in diesen Konstruktionen keine Rolle. Zweitens werden solche Kinder und Jugendliche als ein Faktor benannt, der religiöse Vielfalt konturiert. Säkulare Einstellungen und Ansichten gehören für die Interviewten damit zum Begriff religiöser Vielfalt.

Schließlich finden sich noch sog. unbestimmte Differenzen. In diesen Fällen wird eine nominale Kategorie zur Abgrenzung eines religiös relevanten Sachverhalts herangezogen, ohne dass klar ersichtlich ist, was der Antipode dieser Differenz ist. Das Gegenüber der Unterscheidung bleibt unbestimmt, obwohl die Aussagelogik anzeigt, dass ein Unterschied angenommen wird.

"Auch beim Thema Tod und Sterben in der Klasse Sieben. Die Frage danach, warum sie glauben, dass

Menschen sterben müssen. Das war ganz unterschiedlich, wie Schüler sich das erklären, was der Sinn des

Lebens ist und so. Und ich finde das total spannend, wenn Schüler darüber ins Gespräch kommen. Und

ich denen so ein bisschen dann Impulse gebe, was die christliche Seite angeht. Das ist leider oft sehr weit

weg von denen. Ja, wie Schüler das so sehen" (SIE_011_maxqda, Pos. 3).

Im vorliegenden Beispiel steht die christliche Haltung zu Tod und Sterben für etwas Besonderes, das eigens erwähnt wird. Offensichtlich ist sie "sehr weit weg“ von den Haltungen der Schülerinnen und Schüler bei diesem Thema. Gleichzeitig bleibt das, was dieser christlichen Haltung gegenübersteht, unterbestimmt. Es wird deutlich, dass eine Differenz - im gegebenen Fall zur christlichen Position -, nicht jedoch, worin diese besteht.

\subsection{Religiöse Vielfalt entlang positioneller, stilistischer, kontextueller und biografischer Aspekte}

Alternativ zur eben beschriebenen Heuristik finden sich Passagen in den Interviews, die religiöse Vielfalt anhand positioneller, stilistischer, kontextueller und biografischer Aspekte konstruieren, wie sie sich im oben beschriebenen Modell Rudolf Englerts finden. Insofern sich zu allen vier Dimensionen Passagen aus den narrativen Teilen der Interviews finden lassen, ist sichergestellt, dass diese Heuristik nicht durch die Interviewten induziert wurde.

Bei positionellen Aspekten handelt es sich um weltanschauliche Positionen, aus denen heraus sich Unterschiede speisen und die somit zu religiöser Vielfalt beitragen. Solche Aspekte beziehen sich in der Regel auf einen konkreten Sachverhalt, den man so oder so denken kann, dem man zustimmen kann oder nicht, bzw. dem man sich verbunden fühlt oder nicht. Religiöse Vielfalt wird demnach durch die Präsenz verschiedener Ausprägungen dieses Sachverhalts bedingt.

"Also ich finde sogar, dass man relativ schnell was rausspürt vom Gottesbild. Also wenn ich jetzt... Also und zwar deswegen, weil... Wenn dann Gott zum Beispiel so thematisiert wird, dass Gott ja auch will, dass man sich zum Beispiel an Gesetze hält oder an Regeln hält, dann wird aktiv schon relativ schnell spürbar, was da jetzt für ein Gottesbild auch dahinterstecken kann. Und das ist natürlich immer auch irgendwie so eine Mischung. Also ein anderes, ein Gottesbild, was mir auch ganz oft begegnet, das ist ebenso ein allgemein lieber Gott, der dann eben alle Menschen liebt und so und den man sich dann eben so, auch so lieb wünscht" (TUE_011_maxqda, Pos. 89).

Im Beispiel ist es das Gottesbild, das innerhalb der Lerngruppe unterschiedlich ausgeprägt ist. Konkret wird zwischen einem Gott, der Regeln und Gesetze einfordert, und einem Gott, der bedingungslos liebt, unterschieden. Dabei spielt der religiöse oder konfessionelle Hintergrund keine Rolle für die Differenz. So geht es in der Passage des Interviews, aus dem dieser Ausschnitt stammt, um die Schülerinnen und Schüler der Oberstufe allgemein. Eine konkrete religiöse Tradition wird in der gesamten Passage nicht angesprochen. Religiöse Differenz wird damit quer zu den nominalen Religions- oder Konfessionskategorien entlang inhaltlicher, d. h. positioneller Aspekte konstruiert. 
Gleiches findet sich auch entlang stilistischer Aspekte, d. h. der Art und Weise, wie Religiosität gelebt bzw. praktiziert wird. Ein einschlägiges Beispiel wurde oben bereits unter dem Aspekt der intra-religiösen Differenz angeführt, was andeutet, dass sich die nominalen Kategorien und hier beschriebenen nicht gegenseitig ausschließen:

„Es kommt immer darauf an, wie religiös die Schüler jeweils auch sind. Wenn die Muslime nicht religiös sind, dann sind die halt wie alle anderen auch. Wenn sie religiös sind, dann kommt es wieder ein bisschen drauf an, was sie sind, also wie streng" (TUE_006_maxqda, Pos. 59-60).

Es ist die Strenge der Religiosität, die den Unterschied macht, d. h. wie streng der Islam und seine Normen interpretiert werden. Die Differenz liegt somit nicht in der Sache selbst, wie beim positionellen Aspekt, sondern in der Umsetzung derselben Sache in die Wirklichkeit.

Ein dritter Aspekt dieser Heuristik ist der Kontext, aus dem religiöse Vielfalt resultiert. Zu diesem Kontext gehört das Geschlecht ebenso wie die Herkunft. Letzteres wird im folgenden Beispiel deutlich: "In der Berufsschule haben wir Moslems. [...] Da ist es tatsächlich aber eher abhängig davon, woher die kommen. Also deutsche Moslems haben genauso wenig, können genauso wenig sagen über ihren Glauben wie die deutschen Christen. Allerdings, wenn wir Flüchtlinge haben, für die spielt der Glaube, der lebt noch. Da wird dann auch ganz klar erklärt" (TUE_004_maxqda, Pos. 30).

Wiederum geht es um die Auskunftsfähigkeit der muslimischen Schülerinnen und Schüler. Allerdings wird die Varianz derselben nicht über den stilistischen Aspekt konstruiert, wie im Beispiel davor, sondern über die Herkunft, indem „Deutsche Muslime“ von muslimischen „Flüchtlingen“ unterschieden werden. Dabei verweist die Aussagelogik darauf, dass es nicht die Fluchterfahrung ist, die besagte Varianz erklärt, sondern das Aufwachsen im religiösen Klima der Länder, aus denen diese Schülerinnen und Schüler fliehen mussten.

Der vierte von Englert formulierte Aspekt religiöser Vielfalt ist der biografische (s. o.). Auch dieser findet sich in den Interviews. Das folgende Beispiel ist einschlägig.

"Also sie hat mir gesagt, woran sie glaubt. Und das ist alles unglaublich viel mit ihren eigenen Lebensgeschichten immer verbunden gewesen. Und dann hat sich ein Schüler gemeldet, der aus Syrien kommt, ein Flüchtling, und der hat gesagt, [...] für ihn spielt Glaube eine riesige Rolle, weil er auf der Flucht seinen Bruder verloren hatte und da hat er einfach wirklich so ganz viel beten müssen, war super sauer auf Gott, aber gleichzeitig hat ihm irgendwie der Glaube auch Kraft gegeben und das hat ihn wirklich, das gibt ihm Kraft" (TUE_004_maxqda, Pos.8).

Wiederum wird auf die Flucht verwiesen, dieses Mal ist es aber die persönliche Fluchterfahrung, die den Ausschlag gibt. Diesem syrischen Jugendlichen wird eine Schülerin gegenübergestellt, deren konkreter Glaube ebenfalls eng mit ihren „Lebensgeschichten“ verwoben ist. In beiden Fällen sind es somit die individuellen biografischen Erfahrungen, die sich im Glauben niederschlagen und Unterschiede verursachen.

Eine fünfte Konstellation, mit der sich die vier Englertschen Aspekte hätten erweitern lassen, gibt das empirische Material nicht her. Sämtliche relevanten Kategorien lassen sich positionellen, stilistischen, kontextuellen und biografischen Aspekten restlos zuordnen.

\section{Diskussion}

Dieser Beitrag fragt nach den Subjektiven Theorien von Religionslehrpersonen zu religiöser Vielfalt auf der Grundlage von 22 problem-zentrierten Interviews. Als Ergebnis der inhaltsanalytischen Untersuchung mit formal-strukturierendem Schwerpunkt (Schreier, 2014) können zwei Heuristiken festgehalten werden, gemäß denen die Lehrkräfte besagte Vielfalt konstruieren. Die erste Heuristik greift auf die aus dem Religionsdiskurs bekannten nominalen Kategorien zurück, die zweite auf unterschiedliche Dimensionen, entlang derer sich Religion ausdrücken kann. 
Der Rückgriff auf die nominalen Kategorien des Religionsdiskurses konnte erwartet werden. Sie sind nicht nur im Alltag gebräuchliche Kategorien religiöser Zuschreibung, sondern auch die Grundlage der wenigen vorliegenden empirischen Einsichten in derartige Subjektive Theorien (z. B. Pohl-Patalong et. al., 2016). Seine Brisanz gewinnt dieser Befund aus der Problematisierung derartiger Kategorisierungen in der jüngeren religionsdidaktischen Diskussion (Kilchsperger, 2015; Koch, 2021). Demnach entsprechen derartige stereotype Zuschreibungen weder der empirisch wahrnehmbaren Wirklichkeit noch entsprechen sie dem aktuellen Problembewusstsein der kulturwissenschaftlichen und postkolonialen Forschung (z. B. Cotter \& Robertson, 2016). In dieser Hinsicht könnte der Heterogenitätsdiskurs mit seinem Ruf nach Selbstaufklärung und stetem Hinterfragen der im eigenen Tun reproduzierten Wirklichkeit einen produktiven Impuls liefern (z. B. Grümme, 2018).

Gleichzeitig verweist besagte Heuristik darauf, dass religiöse Vielfalt im vorfindlichen Religionsunterricht nicht auf das religiöse Feld beschränkt bleibt. Mit der trans-religiösen Kategorie wird der Tatsache Rechnung getragen, dass auch säkulare Einstellungen und Haltungen zur Diversität in diesem Fach gehören. Ist Letzteres hinreichend bekannt, bestätigt die vorliegende Analyse den Befund, dass Lehrkräfte Säkularität als eine Facette religiöser Vielfalt einstufen (Pohl-Patalong et al. 2016, S. 30-31) und entsprechend nominal kategorisieren. Dass ihnen hierfür kein etablierter Begriff zur Verfügung steht, unterstützt nochmals die Vermutung, dass sich besagte Heuristik dem Religionsdiskurs verdankt.

Die zweite Heuristik zeigt, dass man religiöse Vielfalt auch ohne die herkömmlichen Kategorien des Religionsdiskurses rekonstruieren kann. Inwiefern die konkreten Aspekte induktiv aus den Daten erwachsen sind oder deduktiv in die Daten hineingelesen wurden, kann trefflich diskutiert werden. Auf der einen Seite kannten die Analysierenden das Modell Rudolf Englerts und waren mit seinen vier Dimensionen vertraut. Auf der anderen Seite entstanden die Codes und Kategorien in einem induktiven Verfahren, bei dem die Englertschen Dimensionen zwar bekannt, in jedem einzelnen Fall jedoch vom Textsegment auf ihre Triftigkeit hin im Analyseteam begründet werden mussten. Außerdem finden sich viele der Passagen, die diesen Dimensionen zugeordnet wurden, in den narrativen Einstiegen der Interviews, in denen der Interviewleitfaden noch keine lenkende Wirkung entfalten konnte. Wahrscheinlich beschreibt eine abduktive Logik den Sachverhalt am besten, gemäß dem das Englertsche Modell eine viable Lösung für die Systematisierung der empirischen Beobachtungen geliefert hat (Ziebertz, Heil \& Prokopf, 2003, S. 25-29). Das bedeutet auf der einen Seite, dass die konkreten Benennungen der vier Dimensionen ohne die Kenntnis besagten Modells eventuell anders gelautet hätten. Gleichzeitig bestätigt es aber die empirische Validität dieses Modells.

Letzteres erscheint uns für die Aus- und Weiterbildung von Religionslehrpersonen weiterführend. Die zweite Heuristik bietet nicht nur eine zum Weltreligionen-Paradigma alternative Möglichkeit, religiöse Vielfalt zu konstruieren, sondern kann auch im Sinn einer ",aufgeklärten Heterogenität" (Grümme, 2018) fruchtbar gemacht werden. Mit dem positionellen, stilistischen, kontextuellen und biografischen Aspekt bietet sie eine zugängliche Systematik, die in Lehre und Weiterbildung leicht kommuniziert werden kann. Und dass die Subjektiven Theorien der von uns befragten Lehrpersonen diese Systematik zwar implizit beinhalten, die Lehrpersonen jedoch nirgendwo bewusst und explizit auf sie zurückgreifen, deutet an, dass hier noch nicht gehobenes Bildungspotenzial liegt. Besagte Systematik könnte dazu beitragen, die Kompetenz weiterzuentwickeln, religiöse Vielfalt wahrzunehmen und mit ihr umzugehen, indem Erfahrungen und biografische Ereignisse aufgenommen werden, die nicht auf den ersten Blick schon religiös sind.

Abschließend sei noch kurz auf die Limitationen der vorliegenden Analyse eingegangen. Insofern es sich hier um eine formal-strukturierende Analyse handelt, die vor allem die Struktur des Materials herausarbeiten will, wurde die Reichhaltigkeit dieses Materials sicher noch nicht ausgeschöpft. So könnte anhand der explikativen Version der Inhaltsanalyse (Schreier, 2014) z. B. stärker die kulturelle Verwo- 
benheit der religiösen Kategorien, wie sie von den Lehrpersonen verwendet werden, herausgearbeitet werden. Ebenso könnte eine Inhaltsanalyse durch Extraktion (Schreier, 2014) pointierter und detaillierter die Übereinstimmungen und Unterschiede der Subjektiven Theorien der Lehrpersonen zu religiöser Vielfalt mit der vorfindlichen religionspädagogischen Diskussion herausarbeiten. Und mit der evaluativen Version dieser Methode (Schreier, 2014) könnten z. B. die Unsicherheiten und Suchprozesse der Befragten in der Entwicklung ihrer Subjektiven Theorien stärker in den Blick genommen werden. Alles das würde aber den Rahmen des vorliegenden Beitrags sprengen und verweist auf die Reichhaltigkeit des Materials, das es in weiteren Analyseschritten zu heben gilt.

\section{Literaturverzeichnis}

Aslan, Ednan (2017). Die Erziehung muslimischer Kinder zu Pluralitätsfähigkeit. In Yaşar Sarıkaya \& Franz-Josef Bäumer (Hg.), Aufbruch zu neuen Ufern. Aufgaben, Problemlagen und Profile einer Islamischen Religionspädagogik im europäischen Kontext (S. 15-32). Münster: Waxmann.

Büttner, Gerhard; Mendl, Hans; Reis, Oliver \& Roose, Hanna (Hg.) (2018). Heterogenität im Klassenzimmer. Babenhausen: Verlag LUSA.

Cotter, Christopher R. \& Robertson, David G. (Eds.) (2016). After world religions. Reconstructing Religious Studies. London: Routledge.

Englert, Rudolf (2002a). Skizze einer pluralitätsfähigen Religionspädagogik. In Friedrich Schweitzer, Ulrich Schwab, Hans-Georg Ziebertz \& Rudolf Englert (Hg.), Entwurf einer pluralitätsfähigen Religionspädagogik (S. 87-106). Gütersloh \& Freiburg: Gütersloher Verlagshaus \& Herder.

Englert, Rudolf (2002b). Dimensionen religiöser Pluralität. In Friedrich Schweitzer, Ulrich Schwab, Hans-Georg Ziebertz \& Rudolf Englert (Hg.), Entwurf einer pluralitätsfähigen Religionspädagogik (S. 17-50). Gütersloh \& Freiburg: Gütersloher Verlagshaus \& Herder.

Englert, Rudolf (2008). Religionspädagogische Grundfragen. Anstöße zur Urteilsbildung (2. Auflage). Stuttgart: Kohlhammer.

Grümme, Bernhard (2014). Bildungsgerechtigkeit. Eine religionspädagogische Herausforderung. Stuttgart: Kohlhammer.

Grümme, Bernhard (2017). Heterogenität in der Religionspädagogik. Grundlagen und konkrete Bausteine. Freiburg: Herder.

Grümme, Bernhard (2018). Auf dem Weg zu einem Paradigmenwechsel in der Religionspädagogik? Skizzen zu einem Konzept Aufgeklärter Heterogenität. Praktische Theologie, 53(2), 74-78.

Kilchsperger, Johannes (2015). Religionskundliches Lernen: Kulturelle Zugänge und religiöse Konzepte. In Sophia Bietenhard, Dominik Helbling \& Kuno Schmid (Hg.), Ethik, Religionen, Gemeinschaft. Ein Studienbuch (S. 203-212). Bern: hep, der Bildungsverlag.

Kindermann, Katharina \& Riegel, Ulrich (2016). Subjektive Theorien von Lehrpersonen. Variationen und methodische Modifikationen eines Forschungsprogramms. Forum Qualitative Sozialforschung, 17(2), Art. 1. https://doi.org/10.17169/fqs-17.2.2486

Klinkhammer, Gritt \& Neumaier, Anna (2020). Religiöse Pluralitäten - Umbrüche in der Wahrnehmung religiöser Vielfalt in Deutschland. Bielefeld: transcript.

Koch, Anne (2021). Dringender Nachholbedarf. Der Anachronismus ,Weltreligionen' in der Didaktik religiösen Pluralismus. Österreichisches Religionspädagogisches Forum, 29(1), 275-291. https://doi.org/10.25364/10.29:2021.1.17

Lehner-Hartmann, Andrea (2014). Religiöses Lernen. Subjektive Theorien von ReligionslehrerInnen. Stuttgart: Kohlhammer.

Mayring, Philipp (2000). Qualitative Inhaltsanalyse. Forum Qualitative Sozialforschung, 1(2), Art. 20. https://doi.org/10.17169/fqs-1.2.1089 
Merk, Samuel (2020). Überzeugungen. In Colin Cramer, Johannes König, Martin Rothland \& Sigrid Blömeke (Hg.), Handbuch Lehrerinnen- und Lehrerbildung (S. 825-832). Bad Heilbrunn: Julius Klinkhardt.

Meyer, Karlo (2019). Grundlagen interreligiösen Lernens. Göttingen: Vandenhoek \& Ruprecht.

Nipkow, Karl E. (1998). Bildung in einer pluralen Welt. Band 1: Moralpädagogik und Pluralismus. Gütersloh: Gütersloher Verlagshaus.

Pohl-Patalong, Uta; Woyke, Johannes; Boll, Stefanie; Dittrich, Thorsten \& Lüdtke, Antonia E. (2016). Konfessioneller Religionsunterricht in religiöser Vielfalt. Eine empirische Studie zum evangelischen Religionsunterricht in Schleswig-Holstein. Stuttgart: Kohlhammer.

Rat der Evangelischen Kirche in Deutschland (Hg.) (2014). Religiöse Orientierung gewinnen. Evangelischer Religionsunterricht als Beitrag zu einer pluralitätsfähigen Schule. Eine Denkschrift des Rates der Evangelischen Kirche in Deutschland. Gütersloh: Gütersloher Verlagshaus.

Reis, Oliver (2021). Unsichtbar, lähmend oder steuernd. Heterogenität als didaktischer Akteur im Unterricht. In Bernhard Grümme, Thomas Schlag \& Norbert Ricken (Hg.), Heterogenität: Eine Herausforderung für Religionspädagogik und Erziehungswissenschaft (S. 137-150). Stuttgart: Kohlhammer.

Rothgangel, Martin (2019). Art. Religionslehrer und -lehrerinnen-Forschung. Das wissenschaftlich-religionspädagogische Lexikon im Internet. https://doi.org/10.23768/wirelex.Religionslehrer und lehrerinnenForschung.200684

Schreier, Margrit (2014). Varianten qualitativer Inhaltsanalyse: Ein Wegweiser im Dickicht der Begrifflichkeiten. Forum Qualitative Sozialforschung, 15(1), Art. 18. https://doi.org/10.17169/fqs-15.1.2043

Schweitzer, Friedrich (2002). Religionspädagogische Theoriebildung im Zeichen der Pluralitätsproblematik. In Friedrich Schweitzer, Ulrich Schwab, Hans-Georg Ziebertz \& Rudolf Englert (Hg.), Entwurf einer pluralitätsfähigen Religionspädagogik (S. 75-85). Gütersloh \& Freiburg: Gütersloher Verlagshaus \& Herder.

Schweitzer, Friedrich (2021). Religiöser Pluralismus und Heterogenität: konkurrierende, komplementäre oder inkommensurable Grundbegriffe der Religionspädagogik? In Bernhard Grümme, Thomas Schlag \& Norbert Ricken (Hg.), Heterogenität: Eine Herausforderung für Religionspädagogik und Erziehungswissenschaft (S. 29-39). Stuttgart: Kohlhammer.

Sekretariat der deutschen Bischofskonferenz [DBK] (Hg.) (2016). Die Zukunft des konfessionellen Religionsunterrichts. Empfehlungen für die Kooperation des katholischen mit dem evangelischen Religionsunterricht. Bonn: Sekretariat der Deutschen Bischofskonferenz.

Simojoki, Henrik (2020). Faktische Beziehungslosigkeit - grundlegende Zusammengehörigkeit. Zur Pluralitätsverarbeitung in Religionspädagogik und Praktischer Theologie. In Bernd Schröder \& Thomas Schlag (Hg.), Praktische Theologie und Religionspädagogik: Systematische, empirische und thematische Verhältnisbestimmungen (S. 103-118). Leipzig: Evangelische Verlagsanstalt.

Trautmann, Matthias \& Wischer, Beate (2011). Heterogenität in der Schule: Eine kritische Einführung. Wiesbaden: VS Verlag für Sozialwissenschaften.

Unser, Alexander (2019). Social inequality and interreligious learning: An empirical analysis of students' agency to cope with interreligious learning tasks. Wien: LIT.

Witzel, Andreas (2000). Das problemzentrierte Interview. Forum Qualitative Sozialforschung, 1(1), Art. 22. https://doi.org/10.17169/fqs-1.1.1132

Ziebertz, Hans-Georg; Heil, Stefan \& Prokopf, Andreas (2003). Gewagte Hypothesen - Abduktion in der Religionspädagogik. In Hans-Georg Ziebertz, Stefan Heil \& Andreas Prokopf (Hg.), Abduktive Korrelation. Religionspädagogische Konzeption, Methodologie und Professionalität im interdisziplinären Dia$\log$ (S. 11-31). Münster: LIT. 